\title{
Fomento del aprendizaje activo y la motivación en el aprendizaje del inglés para fines específicos mediante metodologías de enseñanza centradas en el alumno
}

\section{Ana Sevilla Pavón ${ }^{\mathrm{a}}$, Beatriz Cerezo Merchán ${ }^{\mathrm{b}}$}

a Departament de Filologia Anglesa i Alemanya, Universitat de València. Ana.M.Sevilla@uv.es, ${ }^{b}$ Departament de Filologia Anglesa i Alemanya, Universitat de València. Beatriz.Cerezo@uv.es

\begin{abstract}
This article deals with an educational innovation project around the application of learner-centred methodologies and strategies:gamification and flipped classroom. It was aimed at fostering active learning and motivation among students from the Tourism and International Business Degrees at the Universitat de València. To achieve these goals, students were encouraged to become active protagonists of their own learning processes by means of the exploration of different themes related to their degree. This was done from a socio-constructive and learner-centred perspective through the completion of different tasks around the collaborative creation of digital stories: a WebQuest; a video in the form of a digital story; a self-corrected and collectively-written script; peer-assessment and self-assessment; and oral presentations delivery. The data analysis made it possible to determine the effectiveness of such methodologies and strategies. The results obtained were positive in terms of the students' level of motivation and engagement. Moreover, the goals were achieved, as students' linguistic, communicative and digital competences were enhanced, as well as their creativity and teamworking skills.
\end{abstract}

Keywords: English for Specific Purposes, learner-centred teaching, gamification, flipped classroom, educational innovation.

\footnotetext{
Resumen

Este artículo se centra en un proyecto de innovación educativa en torno a la aplicación de metodologías y estrategias de enseñanza centradas en el alumno ludificación y aula invertida) para fomentar el aprendizaje activo $y$ la motivación del alumnado de los grados de Comercio Internacional y Turismo de la Universitat de València y fomentar sus competencias lingüísticas, comunicativas, digitales, creatividad y capacidad de trabajo en equipo. A tales fines, se invitó a los estudiantes a ser protagonistas de su propio proceso de aprendizaje mediante la exploración de diferentes temáticas relacionadas con su grado desde una perspectiva socioconstructivista y centrada en el estudiante a partir de diferentes tareas en torno a la creación colaborativa de relatos digitales: una WebQuest; un vídeo en forma de relato digital; un guion redactado de manera colectiva $y$
} 
autocorregido; la puesta en marcha de procesos de evaluación por pares y autoevaluación; y la realización de presentaciones orales. El análisis de los datos recabados permitió determinar la efectivadad de dichas metodologías y estrategias. Los resultados obtenidos fueron positivos en cuanto al nivel de motivación e implicación de los alumnos, así como al cumplimiento de los objetivos relativos al desarrollo de diferentes competencias por parte de los estudiantes: lingüisticas, comunicativas, digitales, creatividad y trabajo en equipo.

Palabras clave: inglés para fines específicos, enseñanza centrada en el alumno, ludificación, aula invertida, innovación educativa.

\section{Introducción}

El presente artículo versa sobre el proyecto "Aplicación de metodologías de enseñanza centradas en el alumno y estrategias del aula invertida para fomentar el aprendizaje activo y la motivación en el aula" (ref. UV-SFPIE_RMD15-308327) ${ }^{1}$, que contó con el apoyo del Servicio de formación permanente e innovación educativa de la Universitat de València (UV) durante el curso 2015-2016, en el marco de las ayudas a la innovación y a la renovación de metodologías docentes.

Dicho proyecto giró en torno a la aplicación de metodologías de enseñanza centradas en el alumno: la ludificación y el aula invertida. La aplicación de dichas metodologías pretendía fomentar el aprendizaje activo y la motivación del alumnado de los grados de Comercio Internacional y Turismo. De este modo, se esperaba innovar y enriquecer los programas de enseñanza de y en inglés, con especial atención al inglés para fines específicos; fomentar el desarrollo de competencias lingüísticas, comunicativas y digitales, junto con la creatividad y el trabajo en equipo; examinar el posible valor añadido de estas metodologías y estrategias; y recopilar una selección de materiales a través de RODERIC ${ }^{2}$, el repositorio institucional de objetos de aprendizaje de la UV.

La ludificación o gamificación consiste en el uso de elementos lúdicos y de técnicas de juego en contextos no lúdicos (Werbach \& Hunter, 2012) con el fin de fomentar la participación, motivación, empoderamiento e implicación de los estudiantes, así como el desarrollo de competencias digitales y de resolución de problemas (Kapp, 2012). Este enfoque de aprendizaje fue aplicado en el proyecto findamentalmente al plantear la

\footnotetext{
${ }^{1}$ Las autoras quisieran expresar su agradecimiento a las profesoras participantes en el proyecto: Dra. Elvira M Montañés Brunet, Dra. Ana Isabel Fernández Mesa, Dra. Beatriz Pastor García y Dra. Ana Fernández-Caparrós Turina.

2 El repositorio está disponible en: http://roderic.uv.es/ y un ejemplo de relato digital grabado por los alumnos con apoyo del SFPIE y compilado en el repoistorio de RODERIC está disponible en https://www.youtube.com/watch?v=JDM9PHRjFQM\&feature=youtu.be
}

(c) EY-NC-ND 2016, Universitat Politècnica de València 
narrativa que sirvió como hilo conductor para la realización del trabajo, como se explicará más adelante. Dicha narrativa presentaba a los estudiantes no como tales, sino como jóvenes emprendedores que habían creado una start-up y estaban a punto de lanzar un producto revolucionario e innovador.

Por su parte, el Aula invertida o flipped teaching se basa en una doble inversión de la organización tradicional de los contextos educativos. Por un lado, se invirten los roles profesor-alumno, con lo que el docente pasa a ser un facilitador y guía en el proceso de aprendizaje, mientras que el estudiante desarrolla su autonomía y se hace responsable de dicho proceso de aprendizaje. Por otro lado, se invierten los espacios de casa y del aula. La inversión de espacios implica que la teoría, la preparación y las lecturas de textos o visionado de vídeos se realicen en casa antes de la clase con materiales previamente seleccionados o creados por el docente (Herreid y Schiller, 2013). Ello permite que la práctica y la interacción se realicen en clase, con actividades en grupo, resolución de dudas, discusiones y reflexiones, con el profesor como facilitador del proceso. De este modo, se abren espacios de afinidad en que los estudiantes utilizan una gran variedad de herramientas de manera colaborativa y creativa, poniendo en práctica diversas habilidades y destrezas del siglo 21 para investigar y comunicarse (Gee, 2013). Entre los aspectos positivos del Aula invertida destacan el aumento de la implicación de los estudiantes, el fomento del pensamiento crítico y la mejora de las actitudes de los estudiantes respecto al aprendizaje; la adaptación a distintos ritmos de aprendizaje; y las posibilidades de proporcionar ayuda individualizada a los estudiantes y de adaptar el currículum a las necesidades y espectativas de los alumnos (Fulton, 2012).

Mediante la aplicación de las metodologías anteriormente expuestas, se esperaba convertir a los alumnos en protagonistas de su propio proceso de aprendizaje a través de la exploración de diferentes temáticas relacionadas con su grado de manera socioconstructivista (Vygotsky, 1978) y centrada en el estudiante (O'Neill y McMahon, 2005). Para ello, se les propuso a los alumnos la realización de diferentes tareas en torno a la creación colaborativa de un producto innovador que sería posteriormente presentando ante inversores potenciales en una feria de negocios ficticia, a través de un relato digital y de una presentación oral por grupos.

La recopilación y análisis de los datos mediante cuestionarios, la observación directa, diarios de campo y entrevistas a grupos focales permitieron determinar la efectividad de dichas metodologías y estrategias. Por limitaciones de espacio, en este artículo nos centraremos en el análisis de los resultados cuantitativos y cualitativos del cuestionario de opinión a que respondieron los alumnos de uno de los grupos participantes. Los resultados fueron positivos en cuanto al nivel de motivación e implicación de los alumnos, así como al cumplimiento de los objetivos relativos al desarrollo de diferentes competencias: lingüísticas, comunicativas, digitales, así como creatividad y destrezas de trabajo en equipo.

\section{Objetivos}

Los objetivos del proyecto fueron:

2016, Universitat Politècnica de València

Congreso In-Red (2016) 
a) innovar y enriquecer los programas de enseñanza de y en inglés y, más concretamente, de inglés para fines específicos;

b) fomentar el desarrollo de competencias lingüísticas, comunicativas y digitales en el alumnado, así como la creatividad y el trabajo en equipo;

c) examinar el posible valor añadido de estas metodologías y estrategias;

d) Recopilar una selección de materiales a través de RODERIC, el repositorio institucional de objetos de aprendizaje de la UV.

\section{Desarrollo de la innovación}

El proyecto fue llevado a cabo con un total de 437 estudiantes de edades comprendidas entre los 18 y 25 años y con un nivel B2 o intermedio-alto del MCERL (Consejo de Europa, 2001), sumando los alumnos de: 1) los grupos ARA y GP de Business English III y el grupo ARA de Introducción a la Dirección de empresas; y 2) los alumnos de Comunicación en Lengua Inglesa para Turismo I y II. Sin embargo, debido a limitaciones de espacio, en este trabajo nos centraremos únicamente en los resultados obtenidos a raíz de la aplicación del proyecto con un grupo de 40 alumnos de la asignatura Business English III del Grado en International Business.

Los estudiantes trabajaron en grupos de 4 para llevar a cabo tareas en torno a la creación de relatos digitales sobre temáticas relacionadas con los negocios internacionales y el turismo. Dichas tareas fueron propuestas a partir de los enfoques de ludificación y aula invertida, y se adaptaron a las características específicas de cada asignatura. En el caso de Business English III, la asignatura se divide en sesiones de tres tipos: clases teóricas, clases prácticas y sesiones de laboratorio. Por su parte, las asignaturas de Introducción a la Dirección de Empresas y Comunicación en Lengua Inglesa para el Turismo, las sesiones son de dos tipos: clases teóricas y clases prácticas. El enfoque de clase invertida se aplicó a las clases teóricas en combinación con estrategias de ludificación, mientras que en las clases prácticas y en las sesiones de laboratorio se implementaron diversas estrategias de ludificación.

Las tareas realizadas en el marco del proyecto, en el caso concreto de las asignaturas de Inglés para los Negocios e Introducción a la Dirección de Empresas, giraban en torno a la siguiente narrativa, propuesta dentro de la lógica de la ludificación, entendiéndose narrativa como el aspecto fundamental de la cognición, comprensión y explicación, siendo también la herramienta más importante en la construcción de identidades e historias (Simons, 2007):

Working in groups, imagine you are young entrepreneurs who have started their own small business (you obviously target an English-speaking market). You are about to launch an extremely innovative product which will add significant value to the market. You need to describe your product or technology in detail: introduce your team and the position in the company; briefly show the structure of the company; describe the product's design, features and consumer benefits; describe the product's target market; mention other competing products or technologies (if any); present your strategy for the new product, e.g. a) Branding, packaging, other features (guarantee, etc.), b) Pricing strategy; c) Distribution (What sales outlets and channels will be used?); d) Promotion (Advertising, product launch and sales promotion).

In order to raise funds for the development and distribution on your product, you need to create a 4-minute long digital story and deliver an oral presentation within the general 
theme: "A revolutionary product". These digital stories can be used as advertising tools by the companies themselves. The final digital stories will be shown in a fictitious international business fair in which you'll have to present your videos and answer questions about the product or technology. In the business fair you'll hold a meeting with several investors (your classmates). Each group of investors have 100,000€ and you'd like them to invest in your product so your presentation should be aimed at persuading them that your product will bring huge dividends to investors, stakeholders and shareholders.

Así pues, con esa narrativa como hilo conductor, los alumnos trabajaron en grupos para hacer su propia propuesta de creación de un producto innovador y revolucionario que sería posteriormente presentado ante inversores potenciales. De cara a la presentación, los estudiantes tenían que preparar tanto un relato digital como una presentación oral. A continuación, se describen cada una de las tareas realizadas por los estudiantes para alcanzar dicho fin:

\section{A) Creación de relatos digitales:}

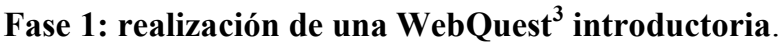

Los alumnos trabajaron en grupos de entre 4 y 5 alumnos para llevar a cabo las diferentes fases, incluyendo la realización de una WebQuest que sirvió para presentar el proyecto. Se entiende como WebQuest una actividad colaborativa de investigación y búsqueda a partir de recursos de Internet, previamente seleccionados por el profesor (Dodge, 1995), para alcanzar un objetivo común. La estructura de la WebQuest propuesta y de creación propia, "Digital Storytelling for International Business", siguió los patrones más comunes en este tipo de actividades: en la sección de "Introducción" se proporcionaba información general en relación al proyecto, incluyendo los objetivos del mismo y el volumen de trabajo requerido; la sección de "Tareas" proporcionaba información específica sobre la realización de estas, incluyendo la organización del trabajo y la temporalización. La siguiente sección, "Proceso", proporcionaba instrucciones más specíficas sobre cómo llevar a cabo las diferentes tareas y proporcionaba acceso a diferentes recursos textuales y audiovisuales; la sección de "evaluación" presentaba la rúbrica de evaluación, junto con una explicación detallada de los criterios de evaluación; y la última sección, "Conclusión", invitaba a la reflexión en torno a lo aprendido. La figura anterior (Figura 1) muestra la pantalla de inicio de la WebQuest introductoria:

\footnotetext{
${ }^{3}$ Disponible en: http://zunal.com/webquest.php?w=255950
} 


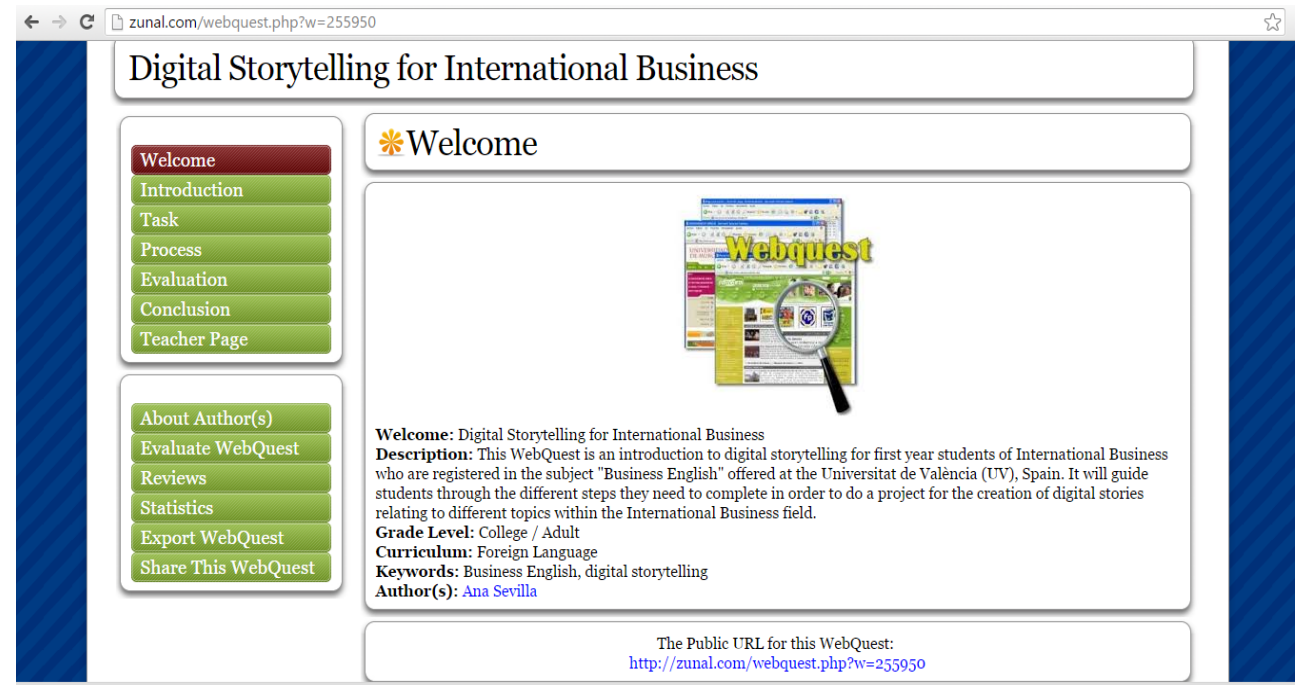

Figura 1. Captura de pantalla de la WebQuest "Digital Storytelling for International Business"

\section{Fase 2: Lluvia de ideas y redacción colaborativa del primer borrador del guion.}

Los diferentes grupos de estudiantes realizaron una lluvia de ideas en relación al producto utilizando la herramienta GoogleDocs, en la cual crearon y editaron de manera simultánea un documento llamado "Ideas for our digital story", tal y como muestra la Figura 2:

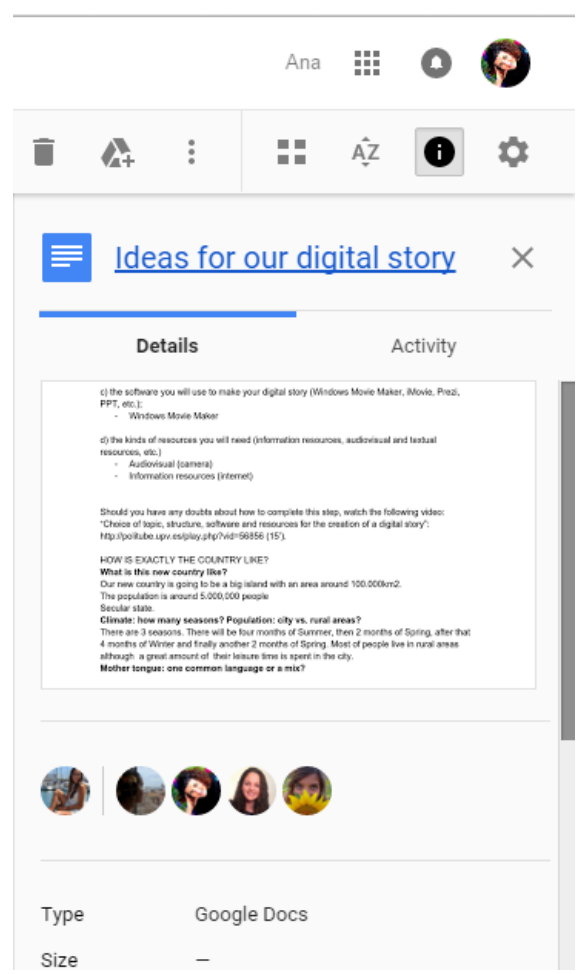

Figura 2. Ejemplo de documento colectivo en GoogleDocs.

(cc) BY-NC-ND 2016, Universitat Politècnica de València 
De entre todos los productos propuestos, los alumnos votaron el que consideraban que más se ajustaba a los criterios preestablecidos y, por ende, el que más posibilidades de éxito podría tener de cara a recibir financiación por parte de los inversores. Una vez alcanzado el consenso en cuanto al producto, los alumnos procedieron a la redacción colectiva y colaborativa del guion. Para ello, utilizaron la herramienta GoogleDocs, que les permitió añadir o eliminar información de manera simultánea, así como reorganizar párrafos y pulir el contenido y la forma en sucesivas versiones. En la redacción del primer borrador de dicho guion, así como en las versiones posteriores, se observó que los estudiantes alternaban y se intercambiaban los papeles de escritor, editor, revisor, líder del equipo y facilitador (Sevilla Pavón, 2015).

Fase 3: Autocorrección del guion y redacción de los sucesivos borradores hasta llegar a la versión final.

Mediante el uso de una plantilla de autocorrección (Sevilla Pavón, 2015), en combinación con la reetroalimentación, proporcionada por las docentes, los alumnos se encargaron de autocorregir sus guiones y generar la versión final de los mismos, que después se convertirían en la base de la narración oral.

Fase 4: Selección de los programas de edición y de los recursos audiovisuales y orden de los recursos en forma de secuencia de vídeo

La elección de los programas de edición de vídeo, que ilustra la figura 3, corrió a cargo de los propios alumnos, quienes tuvieron total libertad para escoger dichos programas según sus preferencias y su nivel de familiarización: Windows Movie Maker, iMovie, etc. Asimismo, se les proporcionaron una serie de enlaces a vídeos tutoriales que explicaban el funcionamiento de los principales programas gratuitos de edición de vídeo. En cuanto a la selección y/o creación de recursos audiovisuales, dicha elección fue el paso previo a creación de una secuencia de vídeo a partir de dichos recursos.

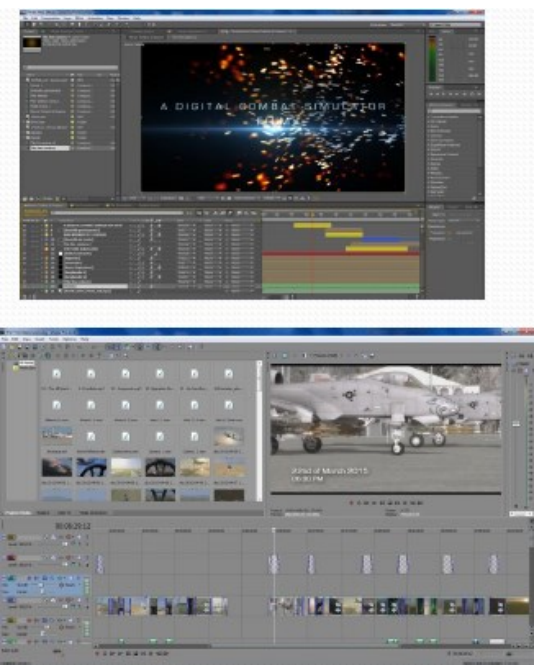

Figura 3. Ejemplos de elección de programas y edición de relatos digitales por parte de los estudiantes. 
Fase 5: Grabación de la narración oral del relato digital y sincronización de los recursos audiovisuales y la voz.

Una vez concluida la secuencia de vídeo, los estudiantes ensayaron la narración oral mediante la lectura en voz alta del guion, prestando especial atención a la pronunciación, a la dicción y a la entonación. Tras varios ensayos, los alumnos grabaron la narración oral y la sincronizaron con los recursos audiovisuales.

Fase 6: Inclusión de subtítulos y banda sonora y finalización de los relatos digitales.

Con el fin de hacer los relatos digitales más accesibles, los estudiantes añadieron los correspondientes subtítulos, de nuevo utilizando programas gratuitos de su elección para tal fin. Del mismo modo, añadieron bandas sonoras e incluso efectos sonoros para reforzar el mensaje transmitido en cada uno de los relatos. Seguidamente, los estudiantes exportaron los relatos digitales en el formato correspondiente, los subieron a la web en modo privado y compartieron los enlaces a los diferentes vídeos través del foro de discusión en línea de Google+. Ello permitió a cada grupo poder ver los vídeos creados por los compañeros.

\section{Fase 7: Visionado y evaluación de los relatos digitales.}

Tras haber subido los vídeos a la web y, habiendo visto los diferentes relatos digitales, los estudiantes procedieron a la evaluación por pares de los mismos a través de la rúbrica de evaluación (Figura 4). Asimismo, también se produjo un intercambio de comentarios y críticas constructivas en el foro de discusión en línea, tal y como muestra la figura 5:

\begin{tabular}{|c|c|c|}
\hline 1) Title: & Score (1-10) & \multirow{6}{*}{$\begin{array}{l}\text { NEW PRODUCT RATING CARD: } \\
\text { (assess ONLY the relevant items } \\
\text { based on the level clarity, } \\
\text { persuasion } \\
\text { and impact) }\end{array}$} \\
\hline $\begin{array}{l}\text { Introduction (team, position, } \\
\text { structure of the company, etc.) }\end{array}$ & & \\
\hline $\begin{array}{l}\begin{array}{l}\text { Design, features and consumer } \\
\text { benefits }\end{array} \\
\text { ber }\end{array}$ & & \\
\hline Feasibility & & \\
\hline \begin{tabular}{|l} 
Pricing strategy \\
\end{tabular} & & \\
\hline \begin{tabular}{|l}
$\begin{array}{l}\text { Distribution (sales outlets and } \\
\text { channels) }\end{array}$ \\
\end{tabular} & & \\
\hline \begin{tabular}{|l} 
Promotion (advertising, \\
product launch and sales \\
promotion)
\end{tabular} & & \multirow{4}{*}{$\begin{array}{l}9-10=\text { Outstanding } \\
6-8=\text { Satisfactory }\end{array}$} \\
\hline $\begin{array}{l}\text { Competing products or } \\
\text { technologies }\end{array}$ & & \\
\hline Strategies: packaging & & \\
\hline Strategies: branding & & \\
\hline $\begin{array}{l}\begin{array}{l}\text { Strategies: other features } \\
\text { (guarantee, etc.). }\end{array} \\
\text { gital }\end{array}$ & & \multirow[t]{2}{*}{$1-3=$ Very poor } \\
\hline Conclusion & & \\
\hline
\end{tabular}

Figura 4. Rúbrica de evaluación del producto. 


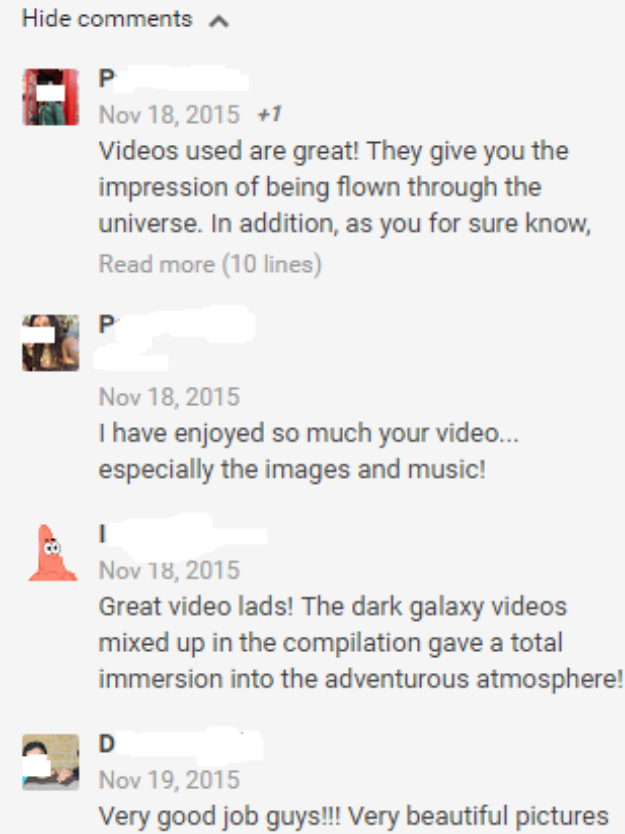

Figura 5. Ejemplo de interacción en el foro de discusión en línea.

\section{B) Preparación y realización de presentaciones orales:}

Fase 1: Preparación del guion de la presentación y de los materiales de apoyo: PowerPoint/Prezi, prototipo del producto, etc.

Tras haber completado los relatos digitales en que se describían las características principales de cada uno de los productos, los estudiantes se centraron en la preparación de la presentación oral. En primer lugar, los estudiantes elaboraron un guion para la presentación oral de 10 minutos. Cada uno de los estudiantes disponía de alrededor de 2 minutos, por lo que las intervenciones habían de estar bien planificadas y ser claras y concisas. Con el fin de hacer la presentación más atractiva y convinciente para los inversores, los estudiantes prepararon materiales de apoyo muy diversos, que iban desde presentaciones de PowerPoint o Prezi hasta elaborados prototipos de los productos.

Fase 2: Realización de la presentación oral en la feria de negocios ante los inversores (el resto de los alumnos).

En la fecha concretada para la celebración de la feria de negocios, cada grupo de estudiantes tuvo la oportunidad de presentar sus respectivos productos ante los inversores, quienes eran sus propios compañeros de clase.

\section{Fase 3: Evaluación a través de la rúbrica de evaluación.}

Al igual que habían hecho con los relatos digitales, los alumnos evaluaron las diferentes presentaciones orales con ayuda de una rúbrica de evaluación que explicaba los criterios de evaluación (Figura 4).

\section{(c) EY-NC-ND 2016, Universitat Politècnica de València}


Fomento del aprendizaje activo y la motivación en el aprendizaje del inglés para fines específicos mediante metodologías de enseñanza centradas en el alumno

\section{Fase 4: Toma de decisiones en grupo respecto a las inversiones}

Una vez que se habían hecho todas las presentaciones, los estudiantes se reunieron por grupos y decidieron en qué producto o productos intevertirían los $100 \quad .000$ euros disponibles para cada grupo de inversores.

\section{Fase 5: Redacción de un informe por parte de cada grupo de inversores.}

Los estudiantes, en sus diferentes grupos, elaboraron y compartieron un informe de inversores en que justificaron los motivos que les habían llevado a invertir en determinado(s) producto(s), junto con la relación detallada de las cantidades invertidas. La Figura 6 muestra un ejemplo de un informe de inversores redactado y compartido por los alumnos en la plataforma Google+.

INVESTORS REPORT
$\begin{aligned} & \text { IN } \\ & \text { Ana Melasa Moderator - Discussion }\end{aligned}$

Figura 6. Captura de pantalla de la Comunidad de Google+ en que se muestra la invitación al concurso y un ejemplo de un informe de los inversores. 
Las tareas anteriormente expuestas fueron complementadas con la celebración de concursos de relatos digitales, la organización de los cuales corrió a cargo de los propios alumnos junto a sus respectivas profesoras. Así, en el mes de diciembre se llevaron a cabo en el Salón de Actos de la Biblioteca Gregori Maians los mencionados concursos con sus correspondientes ceremonias de entrega de premios y despedida de los participantes en el proyecto, con una presencia de alrededor de 190 alumnos en ambos casos. Por un lado, el 14 de diciembre de 2015 se celebró el "2nd International Business Digital Storytelling Contest" en que, además de la proyección de los relatos digitales finalistas, se hicieron las presentaciones orales de los productos más votados, todo ello amenizado por actuaciones de música y baile por parte de los propios alumnos. Una vez celebrados los correspondientes concursos, se hizo un vídeo breve de despedida ${ }^{4}$ Por otro lado, el 18 de diciembre de 2015 tuvo lugar el evento "3rd Digital Storytelling Contest: English for Tourism", en que se proyectaron los relatos digitales finalistas de cada una de las clases, tras una breve presentación de los mismos por parte de los propios alumnos, y se entregaron los premios a los ganadores, previa deliberación por parte del jurado, el cual estaba compuesto por profesores especialistas de las diferentes áreas del conocimiento.

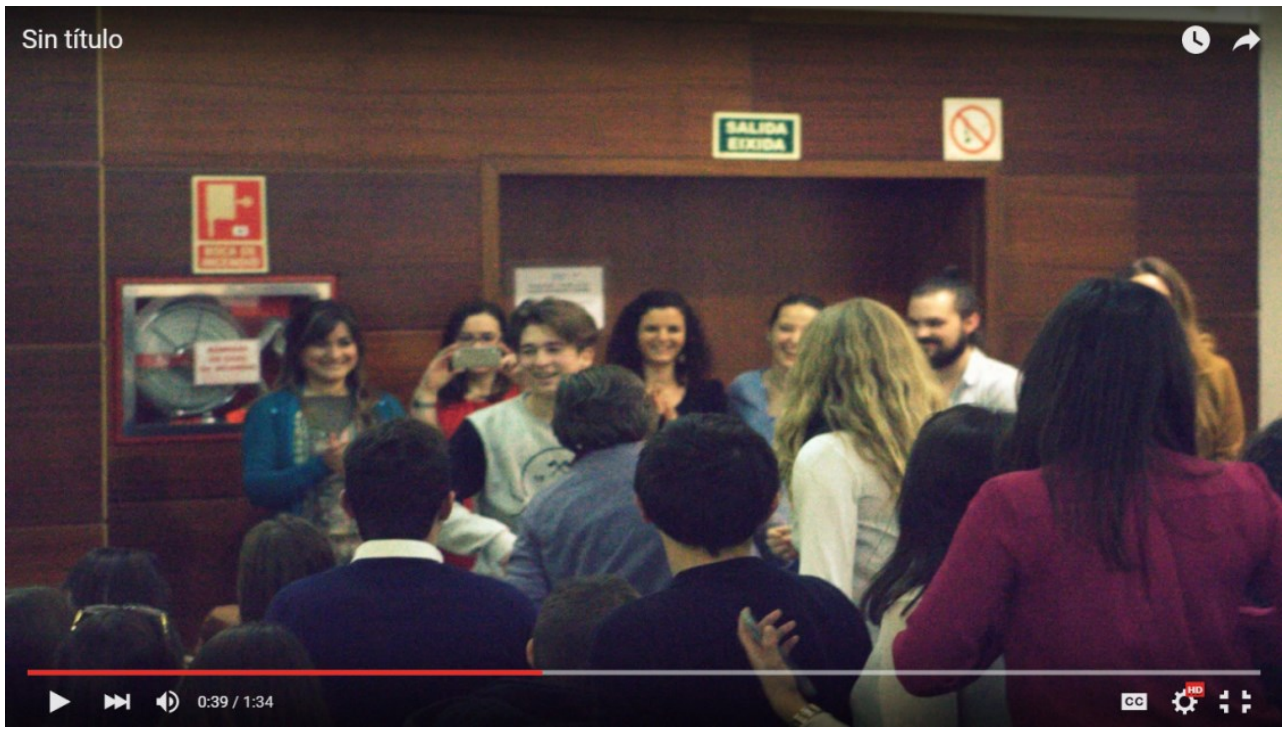

Figura 7. Captura de pantalla del vídeo de despedida elaborado por los alumnos.

\section{Resultados}

La recopilación de datos se realizó mediante la observación directa, un cuestionario de satisfacción y opinión de los participantes, diarios de campo y entrevistas con grupos focales y permitió recabar información respecto a la percepción de los alumnos en cuanto a la efectividad de dichas metodologías y estrategias. Debido a limitaciones de espacio y tiempo, en esta propuesta nos centraremos únicamente en las respuestas de los alumnos en el post-cuestionario de uno de los grupos de alumnos de International Business. El siguiente gráfico resume los resultados de dicho cuestionario de satisfacción y opinión, que obtuvo 40 respuestas por parte de los alumnos de un grupo de International Business en que se

\begin{tabular}{l}
\hline Dicho $\quad$ vídeo, \\
https://www.youtube.com/watch?v=bV7AxTIgM6c\&feature=youtu.be\&ab channel=IsaacMelero
\end{tabular}

\section{(cc) EY-NC-ND 2016, Universitat Politècnica de València}

Congreso In-Red (2016) 
llevó a cabo la innovación. Constaba de 45 preguntas, entre las que se incluían preguntas de selección múltiple (con una escala Likert de 7 puntos) y preguntas de respuesta abierta:

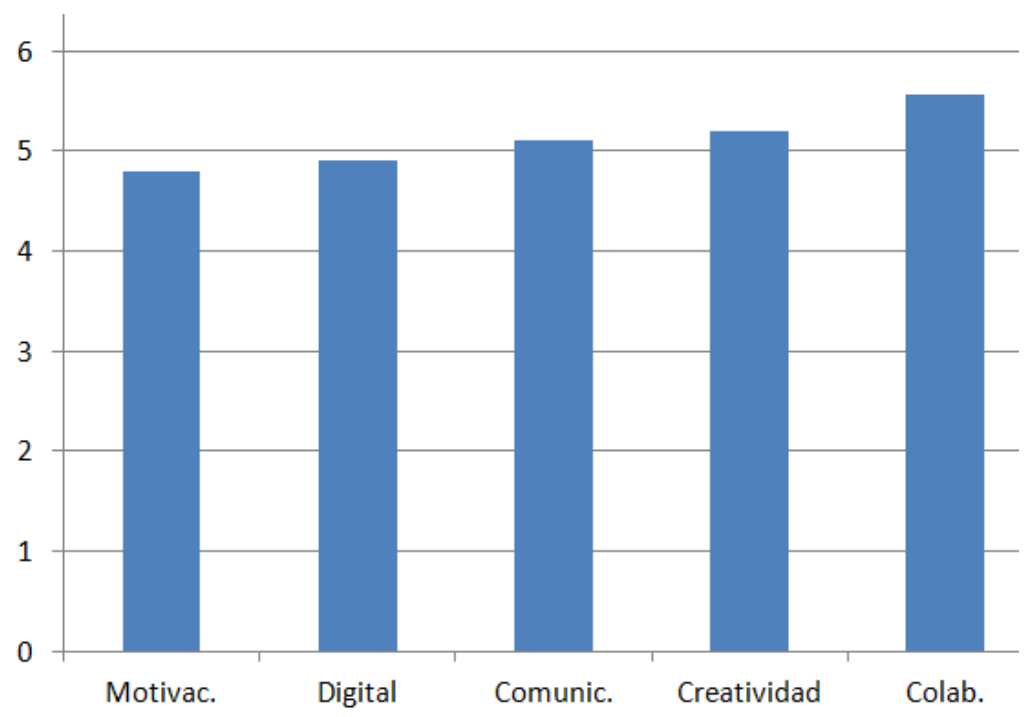

Gráfico 1. Percepción del desarrollo de destrezas de los estudiantes tras el proyecto.

Los resultados fueron, en primer lugar, un alto grado de motivación de los estudiantes y de implicación de los mismos en su propio proceso de aprendizaje, escogiendo el $55 \%$ de los encuestados los valores más altos en relación al poder motivador del proyecto, y afirmando haber sido activos, autónomos y responsables de su aprendizaje. El alto grado de motivación también se apreció en las respuestas abiertas al completar los estudiantes la afirmación: "The project was...":

Respuesta 1. "... at first an unthinkable project, but a satisfaction when it was done".

R2: "... a good way to improve our language skills, communicative skills, team working skills".

R3: "... an exciting experience"

R4: "... a good way to express our ideas".

R5: "... very fun to do".

R6: "... complicated but fun".

R7: "... difficult but funny".

R8: "... amazing".

R9: "... a nice experience, but very time-consuming".

R10: "... completly insane, we are finalists and hopefully we win the "throphy" because I think we did a very good work explaining all the details in a really creative video".

Del mismo modo, según la opinión expresada por los alumnos en las entrevistas y en sus respuestas en los cuestionarios, la realización del proyecto favoreció la competencia digital en el alumnado, mostrándose el $60 \%$ de los encuestados muy de acuerdo en que el proyecto

(cc) EY-NC-ND 2016, Universitat Politècnica de València 
les había ayudado a desarrollar dicha competencia. Estas opiniones favorables también se apreciaron en las respuestas abiertas de los estudiantes, tal y como muestran los siguientes ejemplos de respuestas completando la afirmación "as far as the development of digital skills is concerned, this project was..."

Respuesta 1: "... the perfect fit to unusual technological users".

R2: "... a very proactive activity".

R3: "... helpful since you have to face your digital skills and maybe need to improve them".

R4: "... a useful method to become familiar with digital technology".

R5: ".. an aid to improve our digital skills".

R6: "... challenging".

R7: "... appropriate to improve our digital skills using many internet programs".

R8: "... difficult to prepare but funny and creative".

R9: "... useful for learning how to use technology".

R10: "... good because I improved my digital skills".

Por su parte, las percepciones de los estudiantes también fueron positivas en cuanto al desarrollo de competencias lingüísticas y comunicativas gracias al proyecto. Ello quedó manifiesto en la opinión expresada por el $75 \%$ de los estudiantes, que escogieron los valores máximos al responder a la pregunta relativa a dichas competencias, y también se pudo apreciar en las respuestas abiertas al completar la afirmación "Concerning English language learning, this activity can be considered as...":

Respuesta 1: " an allround activity (speaking, writing, listening)".

R2: "... a good way to improve your speaking and vocabulary".

R3: "... excellent for practicing the language".

R4: "... helpful for students that don't practise English speaking so much".

R5: "... very useful; I've applied the vocabulary we've learned in class and new one".

R6: "... a good way to learn English".

R7: "... a great opportunity to learn business English".

R8: "... good for learning how to communicate if you don't know how to".

R9: "... excepcional!"

R10: "... very useful, since it includes communicating, speaking, listening and understanding (summarizing and thinking) in English.

En cuanto al fomento de la creatividad, el $70 \%$ de los encuestados otorgaron los valores máximos a la misma. Del mismo modo, expresaron opiniones muy favorables al respecto en sus respuestas abiertas en diferentes apartados del cuestionario, particularmente en la pregunta en relación al aspecto que más les había gustado del proyecto, tal y como reflejan las siguientes respuestas:

(cc) EY-NC-ND 2016, Universitat Politècnica de València

Congreso In-Red (2016) 
Respuesta 1: "... showing our own creativity"

R2: "... making the digital story, working as a team, using our imagination to create a new product".

R3: "... creativity".

R4: "... using a creative approach".

R5: "... the creative ideas".

R6: "... that we had a chance to be creative".

R7: "... working in group, creating something with the help of the teacher and the university technology services".

R8: "... that it was interesting, useful and creative".

R9: "... the creative process".

R10: "... learning that all of us have enough creativity to start their business".

Por último, las destrezas de trabajo en equipo fueron las más valoradas por los estudiantes, seleccionando el $80 \%$ de los encuestados los valores máximos de la escala en las preguntas de selección múltiple relativas a dicho aspecto. Además, las afirmaciones de los estudiantes en sus respuestas abiertas corroboraron dicha valoración postitiva , como puede apreciarse en los siguientes ejemplos de respuestas al completar la afirmación "Team working helped me to..":

Respuesta 1: "improve my soft skills".

R2: "... improve my teamworking skills".

R3: "... improve my comunnicative skills".

R4: "... be able to listen to others' opinions and coordinate our work".

R5: "... be more helpful".

R6: "... be more organised".

R7: "... share my ideas and listen and learn about other ones".

R8: "... improve the coordination and speaking skills".

R9: "... to listen to others' opinions and not only my own".

R10: "... find new friends".

\section{Consideraciones finales}

El proyecto aquí descrito forma parte de una inicitativa que recibió el apoyo del Servicio de formación permanente e innovación educativa de la Universitat de València (UV) durante el curso 2015-2016. Su puesta en marcha supuso la aplicación de metodologías y estrategias de enseñanza centradas en el alumno (ludificación y aula invertida) para el fomento del aprendizaje activo y la motivación del alumnado de los grados de Comercio Internacional y Turismo de la Universitat de València a través de un proyecto de creación de relatos digitales en relación a un nuevo e innovador producto que fue presentado en una feria de negocios ante un grupo de inversores, en el caso de los alumnos de comercio

(cc) EY-NC-ND 2016, Universitat Politècnica de València 
internacional, cuya experiencia se ha descrito en el presente artículo con mayor profundidad. Dicho proyecto permitió que los estudiantes dejaran de ser elementos pasivos para convertirse en protagonistas de su proceso de aprendizaje.

La principal limitación de este estudio es el reducido tamaño de la muestra, ya que se presentan únicamente los resultados obtenidos de los cuestionarios finales con un grupo de 40 estudiantes de uno de los grados en que se llevó a cabo la innovación (International Business), de entre los 437 estudiantes de 4 grupos y de dos grados diferentes (International Business y Turismo). En análisis de el resto de los datos recopilados en los diferentes grupos permitirá la obtención de resultados más representativos. Entre las futuras líneas de investigación se incluye el estudio de la interrelación entre determinadas variables, para determinar qué tipo de relaciones se aprecian en este tipo de proyectos entre la motivación, la creatividad, el trabajo en equipo, el desarrollo de competencias comunicativas y lingüísticas, el desarrollo de competencias digitales y qué repercusión podrían tener en el fomento del espíritu emprendedor.

El apoyo institucional y la coordinación entre profesoras de diferentes asignaturas y áreas del conocimiento (inglés para los negocios, inglés para el turismo, dirección de empresas, turismo, etc.) fueron elementos clave para el éxito del proyecto. Los objetivos en relación al fomento del desarrollo de diferentes competencias por parte de los estudiantes: lingüísticas, comunicativas, digitales, creatividad y trabajo en equipo fueron alcanzados, según las opiniones vertidas por los propios estudiantes. También se apreció un alto grado de motivación y una gran implicación de los estudiantes en el proyecto y en su propio proceso de aprendizaje. Del mismo modo, los comentarios y críticas constructivas de los estudiantes servirán para mejorar el proyecto de cara a futuras ediciones del mismo. 
Fomento del aprendizaje activo y la motivación en el aprendizaje del inglés para fines específicos mediante metodologías de enseñanza centradas en el alumno

\section{Bibliografía}

CONSEJO DE EUROPA (2001). Common European Framework of Reference for Languages: Learning, Teaching and Assessment. Cambridge, Cambridge University Press.

DODGE, B. (1995). Some Thoughts About WebQuests. < http://webquest.org/sdsu/about_webquests.html >. [Consulta: 31.03.2016].

FULTON, K. (2012). "Upside down and inside out: Flip your classroom to improve student learning". Learning \& Leading with Technology, Volume 39, Issue 8, p. 12-17.

GEE, J. P. (2013). The Anti-Education Era: Creating Smarter Students Through Digital Learning. New York. Palgrave MacMillan.

HERREID, C. F. \& SCHILleR, N. A. (2013). "Case Studies and the Flipped Classroom". Journal of College Science Teaching. Volume 42 Issue 5, p. 62-66. $<$ http://capone.mtsu.edu/vjm/Univ_Service/CRWG_Home/References/CRWG-SPEE-REF-01.pdf> [Consulta: 31.03.2016].

KAPP, K. М. (2012) The Gamification of Learning and Instruction: Game-based Methods and Strategies for Training and Education. San Francisco, CA: Pfeiffer.

O'NEILL, G. AND MCMAHON, T. (2005) "Student-centred learning: What does it mean for students and lecturers". En G. O'Neill, S. Moore y B. McMullin (Eds): Emerging issues in the practice of university learning and teaching I. Dublin: AISHE.

SEVILla PAVÓN, A. (2015). "Examining collective authorship in collaborative writing tasks through digital storytelling". European Journal of Open and Distance Learning, Volume 1: 1-6.

SIMONS, J. (2007). "Narrative, games, and theory" en Game Studies, Volume 7, Issue 1. $<$ http://gamestudies.org/0701/articles/simons $>$ [Consulta: 10.03.2016]

VyGOTSKY, L. (1978). Mind and Society. Cambridge, MA: Harvard University Press.

WERBACH, K. \& hUnTER, D. (2012). For the Win: How Game Thinking Can Revolutionize Your Business. Philadelphia, PA: Wharton Digital Press.

(cc) EY-NC-ND 2016, Universitat Politècnica de València 